\title{
Spectral shift as advanced index for fruit chlorophyll breakdown
}

\author{
Birgit Seifert • Michael Pflanz • Manuela Zude
}

Received: 5 June 2013 / Accepted: 14 October 2013 / Published online: 6 November 2013

(C) The Author(s) 2013. This article is published with open access at Springerlink.com

\begin{abstract}
The decline of fruit chlorophyll is a valuable indicator of fruit ripeness. Fruit chlorophyll content can be nondestructively estimated by UV/VIS spectroscopy at fixed wavelengths. However, this approach cannot explain the complex changes in chlorophyll catabolism during fruit ripening. We introduce the apparent peak position of the red band chlorophyll absorption as a new qualitative spectral indicator. Climacteric fruit (apple: $n=24$, mango: $n=38$, tomato: $n=48$ ) were analysed at different ripeness stages. The peak position and corresponding intensity values were determined between 650 and $690 \mathrm{~nm}$ of nondestructively measured fruit spectra as well as of corresponding spectra of fruit extracts. In the extracts, individual contents of chlorophyll $a$, chlorophyll $b$, pheophytin $a$ and carotenoids were analysed photometrically, using an established iterative multiple linear regression approach. Nondestructively measured peak positions shifted unimodal in all three fruit species with significant shifts between fruit ripeness classes of maximal $2.00 \pm 0.27 \mathrm{~nm}$ (mean \pm standard error) in tomato and $0.57 \pm 0.11 \mathrm{~nm}$ in apple. Peak positions in extract spectra were related to varying pigment ratios $\left(R_{\max }=-0.91\right)$, considering individual pigments in the pool. The peak intensities in both spectral readings, nondestructive and fruit extracts, were correlated with absolute chlorophyll contents with $R_{\max }=$ -0.84 and $R_{\max }=1.00$, respectively. The introduced spectral
\end{abstract}

Parts of this work have been presented at the CIGR Section VI International Technical Symposium on 'Innovating the Food Value Chain'-Postharvest Technology and Agri-Food Processing in Stellenbosch, South Africa, 25-28 November 2012.

B. Seifert $(\bowtie) \cdot$ M. Pflanz $\cdot$ M. Zude $(\bowtie)$

Department of Horticultural Engineering, Leibniz Institute for Agricultural Engineering Potsdam-Bornim (ATB), Max-Eyth-Allee 100, 14469 Potsdam, Germany

e-mail: bseifert@atb-potsdam.de

e-mail: mzude@atb-potsdam.de marker of the apparent peak position of chlorophyll absorbance bears the potential for an advanced information gain from nondestructive spectra for the determination of fruit ripeness.

Keywords Chlorophyll content - Chlorophyll degradation · Chlorophyll spectrum - Climacteric fruits · Fruit ripeness · Nondestructive

\section{Introduction}

Among physiological changes in ripening fruits (Fry 2003), the degradation of chlorophylls provides a reasonable ripeness indicator. Chlorophyll can be nondestructively measured by spectral-optical readings on the intact fruit: The chlorophyll $a$ molecule bound to the chloroplast's thylakoid membrane shows its maximum absorbance at approximately $680 \mathrm{~nm}$ in vivo. In commercial applications, the measured spectral intensity at fixed wavelength positions is used to calculate vegetation indices, which are well correlated with fruit chlorophyll content (Knee 1972; Nattuvetty and Chen 1980; Zude and Herold 2002; Merzlyak et al. 2003). Hereby, the wavelength range around $680 \mathrm{~nm}$ serves as a prominent parameter. However, during ripening, chloroplasts undergo catabolic changes (Gross 1987) that alter both the environmental conditions of the chlorophyll molecule and its molecular structure. As a consequence, variable spectraloptical characteristics of the fruit may be measured. Changes in the characteristics of the chlorophyll absorbance peakhere not only its intensity but also, in particular, its positionhave never been explored in the context of nondestructive fruit chlorophyll analyses.

Why should the spectral-optical detection of detailed physiological processes be of any use for fruit harvest technology? Particularly in climacteric fruits, the maturity 
stage determines their quality and shelf life. Pre-ethylene burst stages need to be detected and differentiated to determine the optimum harvest date and post-harvest treatments. At that point, the chlorophyll content has started to decline, hence the fruit is starting to lose its green colour (Zude and Herold 2002; Giovannoni 2004; Pereira et al. 2005; Freiman et al. 2012; Nogueira et al. 2012), while the content of carotenoids often has not increased yet (Fraser et al. 1994; Cara and Giovannoni 2008). The blush colour caused by anthocyanins, which give fruits their characteristic pink to red colour, on the other hand, results from cultivar-specific responses to growing conditions and does not indicate specific ripeness stages. Consequently, a detailed analysis of spectral-optical readings of chlorophyll breakdown may lead to more specific indicators for fruit ripeness. According to the most recent review by Hörtensteiner and Kräutler (2011), the enzymatic reduction of chlorophyll $b$ to chlorophyll $a$ is an early crucial step in chlorophyll breakdown in fruits. Following dephytylation leads to the separation from the chlorophyllbinding protein and the formation of chlorophyllide $a$ that is transformed into pheophorbide $a$ by demetallation. The next step opens the porphyrin ring structure, leading to the first non-coloured tetrapyrroles. The demetallized phytylcontaining pheophytin $a$ has been identified as being a breakdown product in leaves only, but it is also part of the photosystem apparatus in the thylakoid membrane of any green plant organ (Eijckelhoff and Dekker 1995, 1997). All early (coloured) chlorophyll breakdown products found in senescent or ripening plant tissue can be distinguished according to their absorbance spectra in the red band range, i.e. near the $680 \mathrm{~nm}$-peak that is typical for chlorophyll $a$ (White et al. 1963; Lichtenthaler and Buschmann 2001).

The presented study aimed to explore (a) if the position of the red band peak in nondestructive spectra of fruits changes with declining total chlorophyll content, (b) if different ratios of chlorophylls and catabolites occur, and-given a positive result in the first two questions - (c) if varying peak positions can be explained by varying pigment ratios. We compared nondestructive spectra and wet chemically analysed pigment compositions from three climacteric fruit species (apple, mango, tomato), testing for the universality of the found pattern.

\section{Materials and Method}

\section{Fruit Material}

For our comparative study, nondestructively and wet chemically obtained spectral-optical characteristics were analysed from three species of climacteric fruits: apple (Malus $x$ domestica 'Jonagold') representing pip fruit, mango (Magnifera indica L. 'Kent') as stone fruit, and tomato
(Solanum lycopersicum L. 'Pannovy'), a berry. Three independent data sets were used. For each, fruits from different ripeness stages were selected to ensure a variation of chlorophyll contents. Apples were harvested from a research fruit station near Berlin, Germany, over eight harvest dates between mid-August and end of October 1999 (first four times biweekly, later weekly). At each date, 15 fruits were harvested and measured and 5 fruits were pooled as one sample, resulting in a total of 24 apple samples. Mango fruits $(n=19)$ were harvested at one date in 2007 in South Africa and immediately shipped to Germany, arriving at varying ripeness stages. Unripe fruits were stored for 5 days at room temperature, while slightly riper fruits were analysed immediately. Since the colour appearance varied greatly within each fruit, probes from two opposite sites of one fruit were considered independent samples, thus totalling in 38 mango samples. Tomatoes were grown under greenhouse conditions in Berlin in 2011, and 48 fruits (representing 48 samples) were harvested at one date. The fruits were grouped into the OECD-standardized ripeness classes 1 to 4 (OECD colour gauge 1992), i.e. they represented mature-green to post-breaker pink fruits.

\section{Nondestructive Spectral-Optical Measurements}

From each sample, nondestructively measured spectra in the visible and NIR range were recorded. Apple and tomato spectra were measured in remittance geometry, using photodiode array spectrophotometers as a portable system (Tec5, Tec5 AG, Germany) and a commercialized handheld device (Pigment Analyzer PA1101, CP, Germany), both with $3.3 \mathrm{~nm}$ wavelength resolution (MMS1, Zeiss, Germany). Both systems were held by hand directly against the equatorial region of the fruits, which were covered by a dark fabric, with a source-detector distance of 8-12 $\mathrm{mm}$. The mango spectra were measured in diffuse reflectance mode with $1.0 \mathrm{~nm}$ wavelength resolution, using a laboratory spectrophotometer with a $150-\mathrm{mm}$ integrating sphere (Lambda 950, Perkin Elmer, USA) with $10-\mathrm{mm}$ aperture. The measured region of the fruit was marked in order to use the same tissue area for the wet chemical reference analysis. For all spectral readings, a spectralon standard (Labsphere, USA) was used for referencing the spectra. The dark spectrum was subtracted from the sample and white spectrum. The spectral absorption of chlorophyll was determined by the ratio between measured and injected light intensity. This generates an inverse peak in the red band area, i.e. with low intensity values for high chlorophyll content in the range from 650 to $690 \mathrm{~nm}$.

For all spectra, Savitzky-Golay filtering was applied for smoothing (second polynomial order, frame width 7) followed by a spline interpolation (MATLAB version 7.11.1, The MathWorks, Inc., USA) to rescale all nondestructive spectra to a 0.5 -nm wavelength resolution. 


\section{Pigment Content}

From each sample, the content of chlorophylls and carotenoids were analysed at the time of the harvest by spectrophotometry after wet chemical acetone (apple, mango) or acetone/ethanol (tomato) extraction (Fish et al. 2002). Apples were probed with a cork borer to obtain equal numbers of 1-mm thick discs. From mangos, approx. 2-mm thick discs of $18 \mathrm{~mm}$ diameter were freshly cut from the exocarp. Pericarp samples of tomato were frozen at $-20{ }^{\circ} \mathrm{C}$ before extraction. The fruit tissue, added by a pinch of calcium carbonate, was ground in a defined volume of the according solvent (containing $0.1 \%$ butylated hydroxytoluene) on ice with an electric tissue grinder (Ultra Turrax, Germany). Resulting pigment extracts were cleaned from residue tissue using a glass frit (pore size 3 ) attached to a vacuum pump. The filtrate was subjected to a phase separation in separating funnel by adding distilled water, transferring non-polar pigments like chlorophyll $a$ and $b$, pheophytin $a$ and most of the carotenoids to a non-polar diethyl ether (apple, mango) or hexane (tomato) phase. The absorbance spectrum of the non-polar phase was recorded in cuvettes $(10 \mathrm{~mm})$ in transmittance geometry in $1 \mathrm{~nm}$ (apple, mango) and $0.5 \mathrm{~nm}$ (tomato) resolution (Lambda 950, Perkin Elmer). From the tomato samples, also the absorbance spectra of the remaining polar phase was recorded, potentially containing the watersoluble chlorophyll catabolites chlorophyllide $a$ and pheophorbide $a$. However, the spectra of this phase showed very low signal to noise ratio and was not analysed further.

From the obtained absorbance spectra, single pigment contents were calculated using the iterative multiple linear regression approach (iMLR) described by Pflanz and Zude (2008). This method is based on individual spectral profiles of pigment standards that are fitted to the measured sum spectrum of the extract. It allows the quantification of individual concentrations of chlorophylls, carotenes and xanthophylls. In our study, the reference spectra of laboratory standards from pure chlorophyll $a$, chlorophyll $b$, pheophytin $a$ and beta-carotene were used. In addition, spectral profiles of lutein and violaxanthin (for mango and apple spectra) and of lycopene (for tomato spectra), resolved in either diethyl ether (Pflanz and Zude 2008) or $n$-hexane, were used. Hexane (Roth, $\geq 98 \%$ ) standards were freshly prepared from the beta-carotene (Roth, 471179506) and lycopene (AppliChem, 10020207) pigments. Chlorophyll a (Sigma, batch number 020M5155V), chlorophyll $b$ (Sigma, 061M5155V), pheophytin $a$ (Chemos, DCL2118) and lutein (AppliChem, 10100511) were first resolved in acetone (Roth, $\geq 99.7 \%$, conc. of $20 \mathrm{mg} / \mathrm{l}$ ) and from there transferred to a hexane phase pigments. The absorbance spectra of the standard solutions were measured according to LambertBeers Law with $0.5 \mathrm{~nm}$ resolution (Lambda 950, Perkin Elmer). The device was photometrically calibrated using a certified potassium dichromate solution (Test Solution for Instrument Performance Validation (ISO 9000, GMP, GLP); Perkin Elmer, Inc.; cell number 9404; perchloric acid reference solution: cell number 10388). Final standard concentrations $(10 \mathrm{mg} / \mathrm{l})$ were calculated using extinction coefficients (Lichtenthaler 1987; Lichtenthaler and Buschmann 2001; Scott 2001). The carotenoids were included for a more accurate total pigment modelling; however, their results will not be further analysed here. Pigment content was related to dry mass in mango and tomato and peel area for apple analyses.

The samples of each fruit species were grouped into four classes ('high', 'medium', 'low', 'very low') according to their total chlorophyll content, which was the sum of the measured chlorophyll $a$, chlorophyll $b$ and pheophytin $a$ contents. For each of these three pigments, also its relative proportion in relation to the total chlorophyll pool was calculated, referring to it as pigment composition. One tomato and one apple sample had to be removed from the reference data sets due to uncertain pigment extraction. The apple sample was kept in the nondestructive data sets due to similar spectral values measured on fruits from the same harvest date.

\section{Spectral Analyses}

From each of the nondestructively (nd) and wet chemically (wc) obtained spectra, the wavelength position of maximum chlorophyll absorption at the red peak between 650 and $690 \mathrm{~nm}$ was calculated. While these values were determined from the baseline-corrected wc spectra, the nd spectra were scatter-corrected by dividing each value by the intensity value at $780 \mathrm{~nm}$, where no pigment-related signal occurs. The peak intensity was obtained as the maximum and minimum intensity values of the red absorption maximum in the baseline-corrected (for wc) and scatter-corrected (for nd) spectra, respectively. For the wc spectra of mango and tomato fruits, peak intensity values were corrected for the variability of the analysed tissue dry mass.

The peak position was derived from the zero crossing in the first derivative of the interpolated (nd) and measured (wc) spectra, calculated as the differential quotient of two (nd) and three (wc) adjacent data points, respectively (i.e. resulting into a higher accuracy than the raw spectra). The wet chemically obtained spectra of the mango and tomato datasets had to be smoothed (SavitzkyGolay filter, second polynomial order, frame width 7) before derivation to account for noise in low intensity readings. From three nd apple spectra and six wc tomato as well as one wc mango spectra (very ripe fruits with lowest chlorophyll contents), peaks were too low to determine peak position and intensity. These samples were excluded from the according analyses. 


\section{Statistics}

All statistical analyses (Pearson correlation, analysis of variance (ANOVA), Tukey post hoc test) were run with SPSS statistics version 21.0 (IBM Corp., USA).

\section{Results and Discussion}

\section{Chlorophyll Gradients}

The three cultivars studied showed a steady gradient of total chlorophyll contents, and thus chlorophyll degradation, with contents decreasing to $25.6,7.0$ and $9.3 \%$ of the highest measured value within the apple, mango and tomato data set, respectively. The relative constant decline allowed us to cluster the samples in four equally sized total chlorophyll content classes within each fruit data set (Fig. 1). In apple, the decrease of total chlorophyll content was negatively correlated with harvest date. Hence, we joined the samples from each two consecutive harvest dates into one chlorophyll content class (Fig. 1). In contrast, in mangos, no relation between chlorophyll contents of fresh and stored samples has been found. For tomatoes, the chlorophyll-based classification corresponded in $69 \%$ of the samples to the visually assigned four OECD classes. Absolute amounts of chlorophyll and other pigments depend on fruit species, cultivar, growing conditions and year and are therefore difficult to compare. However, for apple, similar magnitudes of total chlorophyll contents (although unclear if inclusive pheophytin) were found by Knee (1972). The values decreased on tree from 41.0 to $7.0 \mathrm{mg} / \mathrm{m}^{2}$ over a time comparable to the time between our third and last harvest date, when chlorophyll $a$ and $b$ contents declined from 64.9 to $32.7 \mathrm{mg} / \mathrm{m}^{2}$ (Fig. 1). In a study by Kuckenberg et al. (2008) on 'Jonagold' chlorophyll $a$ and $b$ contents declined from 2234 to $7-13 \mathrm{mg} / \mathrm{m}^{2}$ over 16 days of shelf storage. For mango, comparable data are rare in the literature since chlorophyll contents were determined from the mesocarp only (Kienzle et al. 2012), or only carotenoids were of interest (Ajila et al. 2007; Aziz et al. 2012). However, Janave and Sharma (2006) did report chlorophyll contents in the peel of mangos $\mathrm{cv}$. 'Langra', including chlorophyll $a$ and $b$ and pheophytin $a$. For unripe $(191 \mu \mathrm{g} / \mathrm{g})$ and semi-ripe $(116 \mu \mathrm{g} / \mathrm{g})$ fruits, slightly higher contents were found compared to the present results (Fig. 1). Such differences in the 'greener' stages could also hint to the fact that mangos in the present study were harvested at their commercial picking dates and shipped before analyses, resulting in an advanced ripeness stage in the beginning of the experiment. Chlorophyll $a$ and $b$ contents found in tomato samples (Fig. 1) were similar or slightly lower than data from the literature for fruits of different ripeness stages (Kozukue and Friedman 2003; Pflanz and Zude 2008). Well-comparable data given by Gautier et al. (2008) for their ripeness classes B ('immature green') to E ('orange to deep orange') reveal chlorophyll contents (not specified which) declining from about 54 to $18 \mu \mathrm{g} / \mathrm{g}$, which is lower than our data (Fig. 1). However, the cultivar and season effects can explain the differences. Since in all three mentioned tomato references fresh mass-based values were published, $95 \%$ water content were assumed to transform them into the dry mass-based values presented here.

Characteristics of Nondestructive Fruit Spectra

Are Not Constant with Declining Chlorophyll Content

In the nondestructive readings of intact apple, mango and tomato fruits, the red band signals appeared as an inverse peak. During the ripeness-related degradation of total chlorophyll content, from class 'high' to 'very low', the measured intensity at the minimum of this peak was increasing (Fig. 2, upper row), i.e. the actual peak moved upwards (and by this got smaller). This feature of nondestructively measured fruit spectra is well known (Merzlyak et al. 2003; Zude 2003). It provides an estimator for nondestructive chlorophyll quantification used in several commercial systems for fruit analyses (e.g. DA-Meter,

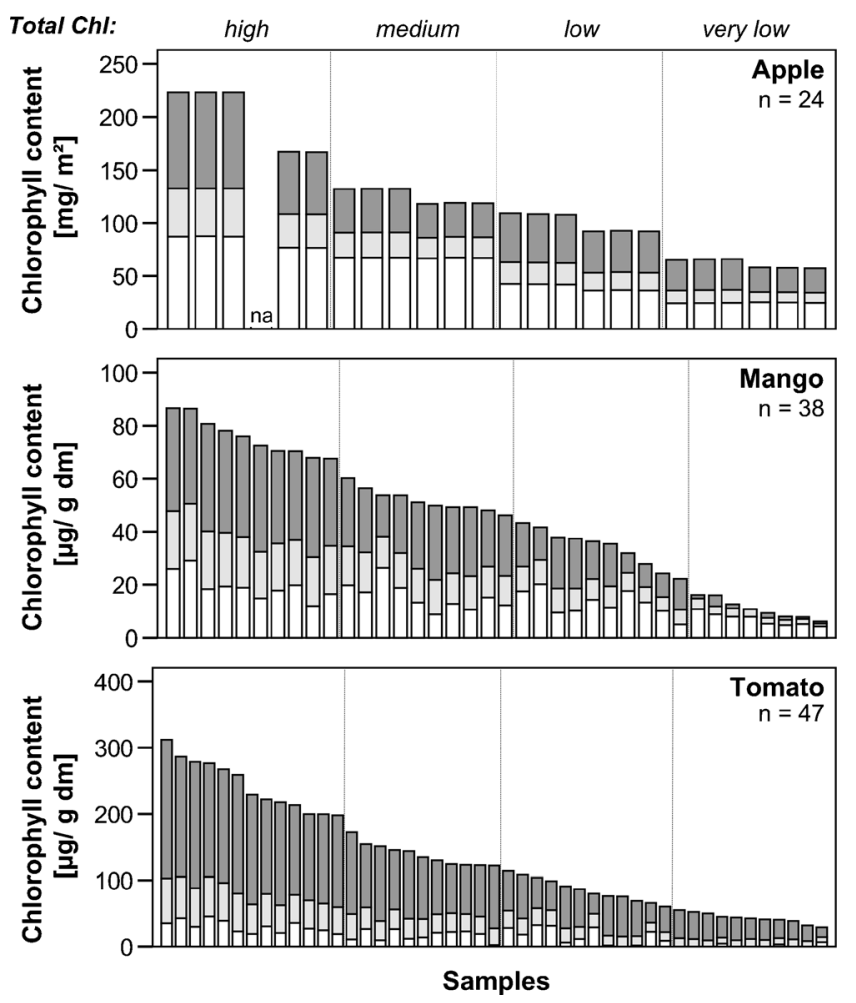

Fig. 1 Samples of the three fruit datasets, presented according to their decreasing total chlorophyll content, i.e. the sum of chlorophyll $a$ (dark grey), chlorophyll $b$ (grey) and pheophytin $a$ (white). Pigment content is related to peel area in apple (data not available $(n a)$ for one sample) and to tissue dry mass $(\mathrm{dm})$ in mango and tomato. Vertical lines separate each the four total chlorophyll content classes (total $\mathrm{Chl}$ ) used in the analyses 
Apple

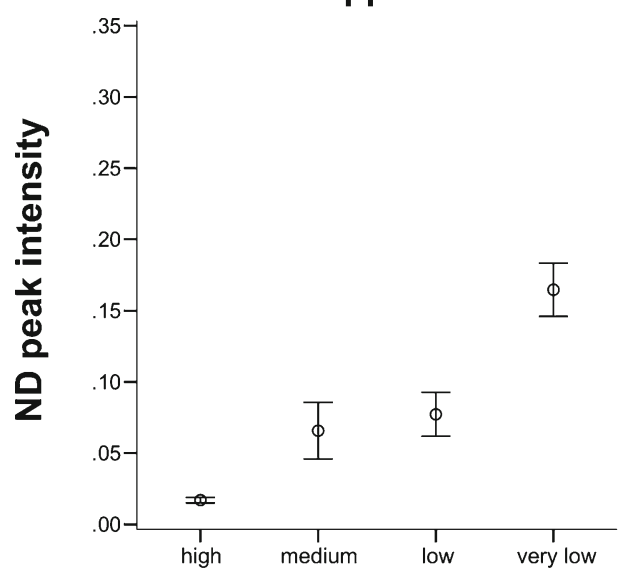

Mango

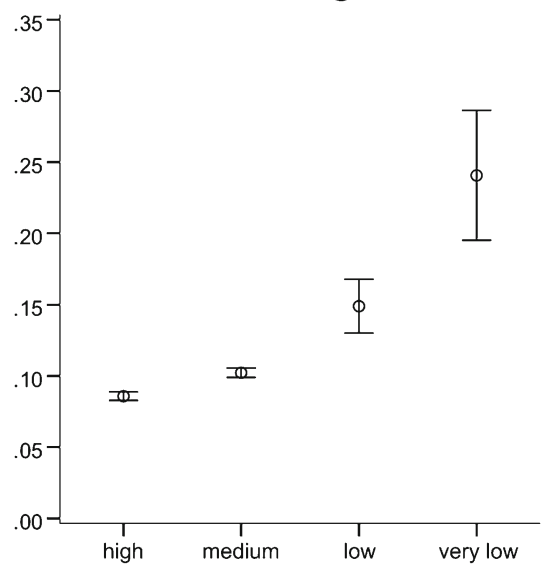

Tomato

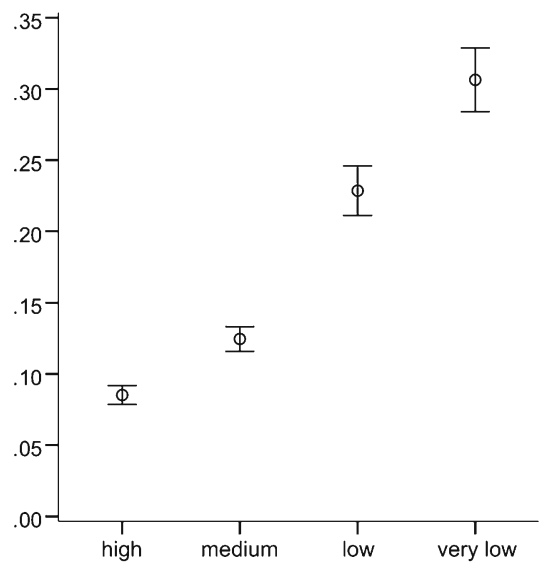

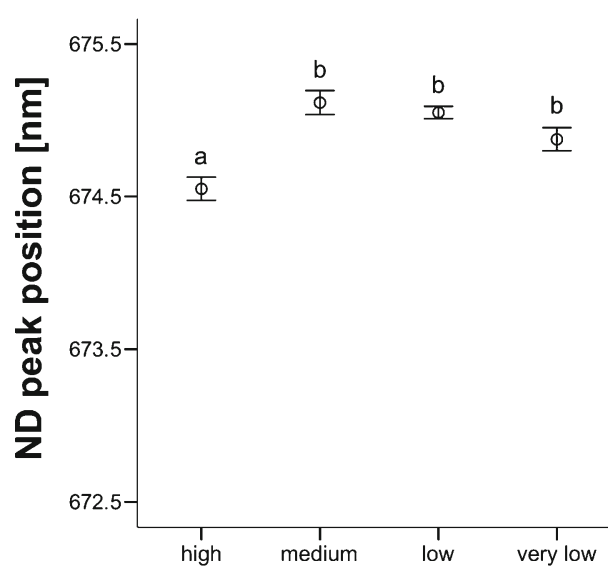
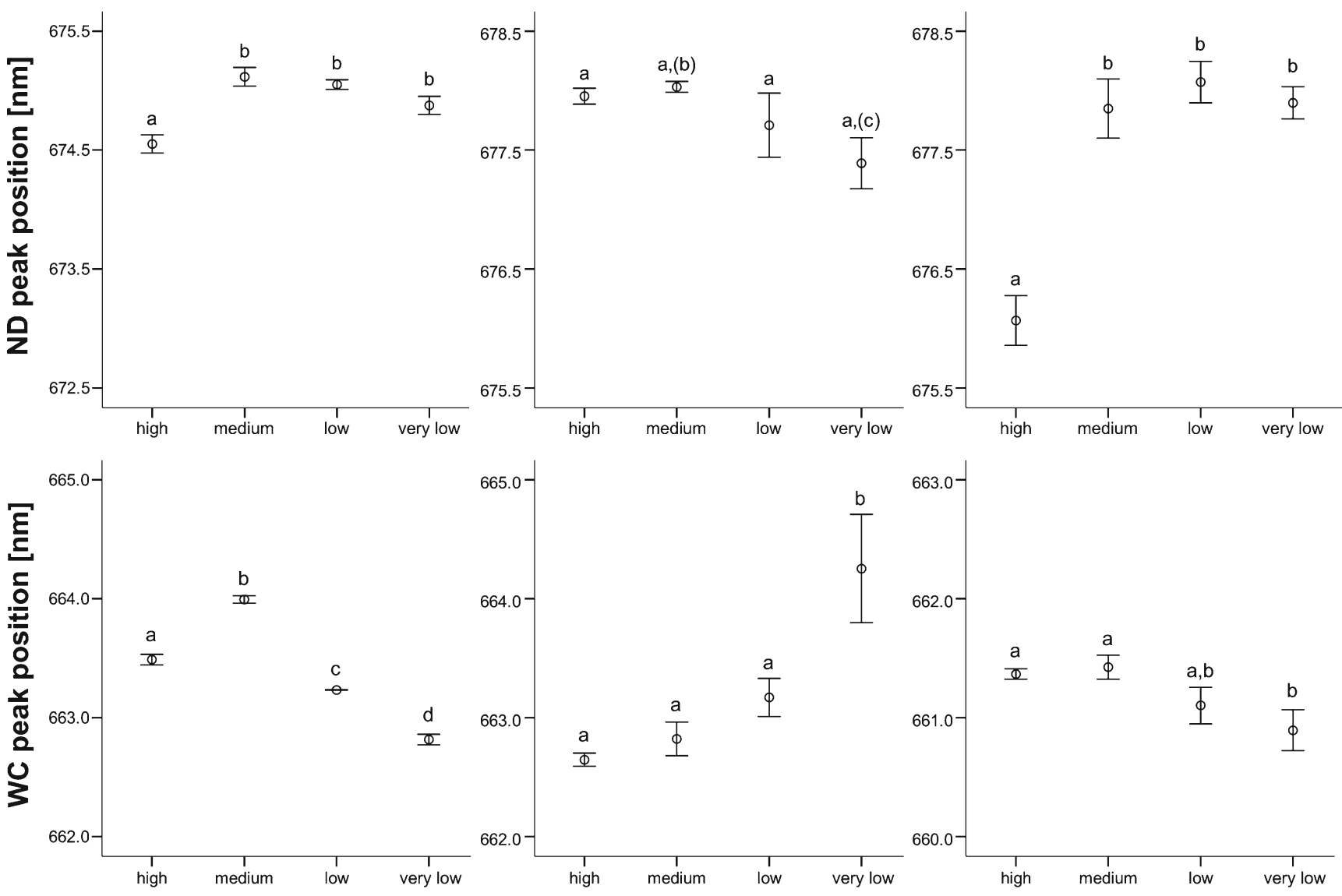

Total chlorophyll content class

Fig. 2 Intensity (upper row) and wavelength position of the red band peak in nondestructive fruit spectra $(N D$, middle row) and in wet chemically derived spectra of fruit extracts (WC, lower row). Bars depict the mean \pm 1 standard error. Different letters indicate significant

Sintéleia, Italy; Pigment Analyzer, CP, Germany) and was confirmed by our data (see below).

More interestingly, the wavelength position of the red band maximum also varied over the declining chlorophyll content. For all three fruit species, a unimodal pattern was found. The differences among chlorophyll classes in their peak positions as tested with ANOVA and Tukey post hoc test. Scale differences on the $y$-axis in the WC spectra result from the different organic solvents used for the pigment extraction

highest wavelength values (i.e. most right positions in the spectra) appeared in samples with medium chlorophyll content, while more left peak positions occurred at higher and lower chlorophyll contents (Fig. 2, middle row). This bidirectional peak shift measured on fresh fruit has, to our 
knowledge, never been described before. Being present in all three fruit species studied, it could point to a general pattern during fruit development.

In the spectra of apple and tomato fruits, the right shift between the first and second chlorophyll class was especially distinct and significant (one-way ANOVA: $F_{\mathrm{A}}=9.50$ [degree of freedom 1, degree of freedom 2=3, 17], $p=0.001 ; F_{\mathrm{T}}=$ 24.29 [3, 43], $p<0.001$, respectively), while only weak and non-significant in mango spectra $\left(F_{\mathrm{M}}=2.57[3,34], p=0.071\right.$; Fig. 2, middle row). In the ripening tomato, the spectral shift calculated from 'high' to 'medium' total chlorophyll content classes was $1.78 \pm 0.27 \mathrm{~nm}$ (mean \pm 1 standard error) and 2.00 $\pm 0.27 \mathrm{~nm}$ between the 'high' and 'low' classes, while it was $0.57 \pm 0.11 \mathrm{~nm}$ between the first two classes in the apple samples. In the more ripe mangos, the shift was minimal and not significant. For the tomato samples, we can link this pronounced shift of the peak position between the deep green fruits of 'high' chlorophyll content and the second highest ('medium') class to the fruits' ethylene status: While all fruits of the first class were still in the pre-ethylene stage, we measured for those of the second class a significantly increased ethylene production (Seifert et al., unpublished data). Hence, at least for this example, we can parallel nondestructively detectable changes in the optical properties of a climacteric fruit to a physiological change that is most relevant in fruit harvest management. Only few pre-harvest data were published on the relation of ethylene production to nondestructive chlorophyll measurements (Zude and Herold 2002; Xie et al. 2009; Solovchenko et al. 2011). These authors correlate the climacteric behaviour in apples and tomatoes to either changes in the red-edge position or multiple wavelength models, respectively. More information exists on colour changes and its interactance with ethylene emission in the post-harvest period (Lang and Hübert 2012). Hence, the described peak shift - if confirmed in further experimentsmay distinguish the pre-climacteric stages from those at the onset of climacteric ethylene production and could be regarded as a promising tool for nondestructive classification of fruit ripeness.

\section{Pigment Composition Varies over Declining Total Chlorophyll Content}

Our approach was to monitor the development of chlorophylls and their breakdown products over the (particularly early) decline of the total chlorophyll content. Further, the pigments' absolute quantities and composition should be linked to spectral measures. The pigment composition found, i.e. the relative proportions of the individual green pigments (chlorophyll $a$, chlorophyll $b$ and pheophytin $a$; each in relation to the total chlorophyll pool), was not constant over the declining total chlorophyll content (Fig. 3). Proportions of chlorophyll $b$ increased (apple, mango) or decreased (tomato) significantly with increasing total chlorophyll content but stayed within a rather narrow range (Fig. 3, middle row). In mango, the proportion of chlorophyll $a$ was significantly declining with declining total chlorophyll content, while it was contrarily for the pheophytin $a$ proportion (Fig. 3, middle column). In apple and tomato, in contrast, chlorophyll $a$ and pheophytin $a$ proportions showed no clear pattern. Nevertheless, in all three fruit species, relative proportions of chlorophyll $a$ and pheophytin $a$ were strongly interrelated: A decrease of the one paralleled an increase of the other, as indicated by a strong negative correlation $\left(R_{\mathrm{A}}=-0.99, R_{\mathrm{M}}=\right.$ $-0.99, R_{\mathrm{T}}=-0.98 ; p<0.001$ for all three fruit species).

These results agree only in parts with the actual knowledge on the chlorophyll breakdown path (Schelbert et al. 2009; Hörtensteiner and Kräutler 2011). According to it, chlorophyll $b$ is reduced to chlorophyll $a$, which is transformed to pheophytin $a$. Thus, the relative proportion of the first two pigments should decline during the course of the chlorophyll degradation process, while the relative proportion of the latter might increase. This pattern we indeed found for the mango samples and to some extent for the apple samples, while in tomato, the chlorophyll pigments behaved differently (Fig. 3). One has to consider here, that the above mentioned breakdown pathway is proposed to be valid for leave tissue only. For fruits, the catabolites chlorophyllide $a$ and pheophorbide $a$ (which we could not extract in sufficient concentrations) are assumed to follow the reduced chlorophyll $a$ instead of pheophytin $a$ (Hörtensteiner and Kräutler 2011). However, a recent study on ripening kiwis (both 'stay-green' and yellowing varieties; Pilkington et al. 2012) revealed the presence of the enzyme pheophytin pheophorbide hydrolase in increasing amounts towards fruit maturity. The enzyme catalyses the transformation of pheophytin $a$ into pheophorbide $a$ (Schelbert et al. 2009; Aiamla-or et al. 2012), suggesting pheophytin $a$ occurring as catabolite also in fruits. Overall, the chlorophyll degradation process is complex and incompletely explored (Hörtensteiner and Kräutler 2011; Saga and Tamiaki 2012), and studies on fruits in particular are underrepresented (Barry 2009). Thus, chlorophyll breakdown processes might vary among the studied fruit types and tissue, considering for example that the stem-derived epidermis and cortex in apple are histologically not comparable with the carpel-derived exocarp and pericarp in mango and tomato.

Varying Pigment Ratios Are Mirrored in the Sum Chlorophyll Peak's Position

Individual chlorophylls, when resolved in organic solvents, can be clearly distinguished according to their peak position in the red band spectral region. The reference standard pigments dissolved in diethyl ether showed peaks at $643 \mathrm{~nm}$ (chlorophyll $b$ ), at $661 \mathrm{~nm}$ (chlorophyll $a$ ) and at $667 \mathrm{~nm}$ 

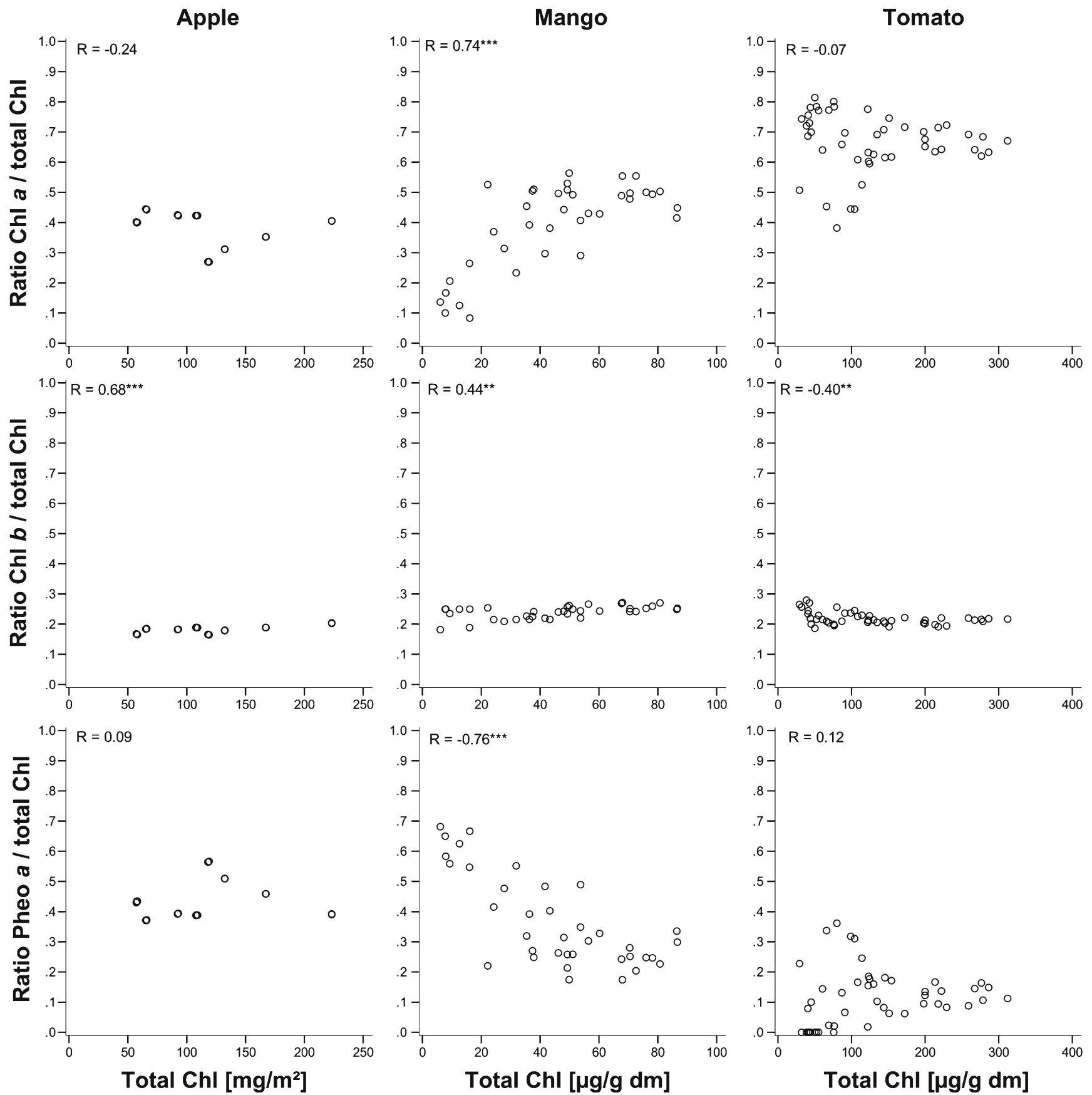

Fig. 3 Proportions of chlorophyll $a(C h l a)$, chlorophyll $b(C h l b)$, and pheophytin $a$ (Pheo $a$ ) in relation to declining total chlorophyll $(\mathrm{Chl})$ content as found in apple $(n=23$; related to peel area), mango $(n=37)$ and

tomato $(n=47$; both related to tissue dry mass $(d m)$ ). Given $R$ values represent the Pearson correlation coefficient with its significance: ${ }^{* *} p \leq$ $0.001 ; * * p<0.01 ; * p \leq 0.05$

(pheophytin $a$ ) (Fig. 4a), which are consistent with the published values (Lichtenthaler 1987). Reference spectra in hexane, as used for the tomato samples, appeared similar (data not shown). In Fig. 4b, c, the influence of different individual pigment ratios on the peak position of the resulting sum spectra is schematized: Under high chlorophyll $a$ proportion, the sum peak lies at lower wavelengths, and under high pheophytin $a$ proportion, the sum peak obviously lies at higher wavelengths. This was confirmed in the fruit extract data: The sum peak shifted to the right (i.e. to higher wavelengths) in samples with higher pheophytin $a$ ratios (i.e. there was a strong positive correlation) and to the left (i.e. negative correlation) with higher chlorophyll $a$ and higher chlorophyll $b$ ratios (Table 1). On the other hand, peak intensity of the sum spectra correlated-as in the nondestructive spectra-highly not only with the total 
$(1: 1: 1)$

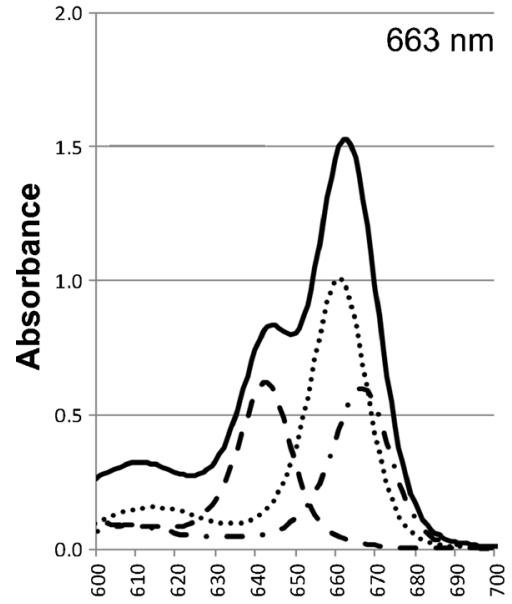

b

Chl.b : Chl.a : Pheo.a

$(0.82: 1.66: 0.52)$

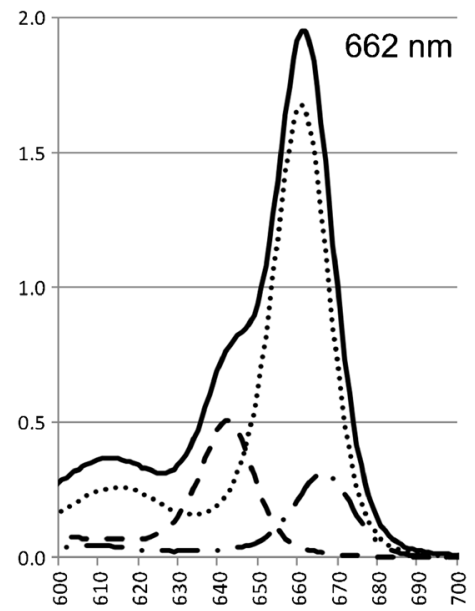

$(0.75: 0.25: 2)$

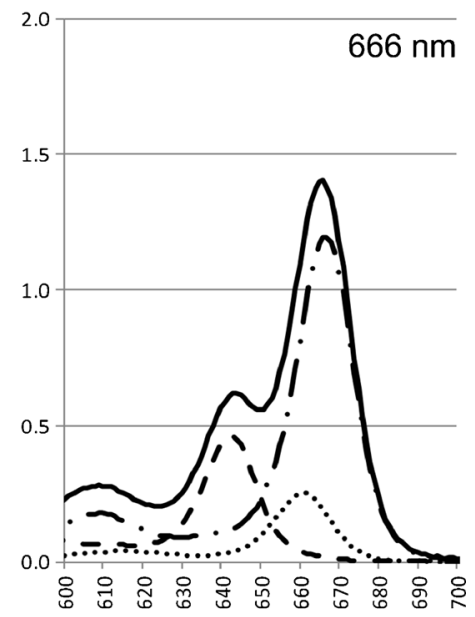

Wavelength $[\mathrm{nm}]$

Fig. 4 Peak position of a sum chlorophyll spectrum (nanometre values) as caused by varying pigment proportions in the non-polar fruit extracts. a Reference spectra of chlorophyll $b$ (Chl.b; dashed), chlorophyll $a$ (Chl.a; dotted) and pheophytin a (Pheo.a; dot-dashed) pigment standards dissolved in diethyl ether $(10 \mathrm{mg} / \mathrm{l}$ each) and resulting sum spectrum (solid line). b Calculated spectra exemplifying a high Chl.a proportion, as measured for a mango sample of the 'high' total chlorophyll content class. c Calculated spectra with a high Pheo.a proportion ('very low' total chlorophyll content class mango sample). For better visibility, sum pigment contents were adjusted to the same value for all three examples $(30 \mathrm{mg} / \mathrm{l})$, thus are not corresponding with the real samples' value chlorophyll content but also with the individual chlorophyll $a$ and chlorophyll $b$ content and to a lesser extent with the pheophytin $a$ content in all three fruit species (Table 1).

Summarizing, the apparent red band peak position in the fruit extracts is related to the relative proportions of chlorophyll $b$, chlorophyll $a$ and pheophytin $a$ and changes in accordance with the individual pigments' spectral properties. Thus, changes in the red band peak position in spectral data allow to a certain extent the extraction of qualitative information of the composition of different chlorophyll and chlorophyll catabolites present in the total chlorophyll pool. On the other hand, the peak intensity gives reasonable estimates for the absolute pigment content. These findings open the possibility to add stronger physiologyrelated information to the commonly applied quantification of the total chlorophyll content that is based on indices from fixed wavelength positions (Merzlyak et al. 2003; Zude 2003; Ziosi et al. 2008).
Table 1 Pearson correlation, $R$, of peak position (PP) and peak intensity (PI) of wet chemical (wc) extract and nondestructive (nd) fruit spectra and the absolute (total chlorophyll (Chl), chlorophyll $a$ (Chl.a), chlorophyll $b$
(Chl.b) and pheophytin $a$ (Pheo.a)), and proportional (e.g. Chl.a: Chl) pigment contents

\begin{tabular}{|c|c|c|c|c|c|c|c|c|c|c|c|c|}
\hline \multirow{3}{*}{$\begin{array}{l}\text { Fruit } \\
\text { Spectra } \\
\text { Variable }\end{array}$} & \multicolumn{4}{|c|}{ Apple } & \multicolumn{4}{|c|}{ Mango } & \multicolumn{4}{|c|}{ Tomato } \\
\hline & \multicolumn{2}{|c|}{ wc } & \multicolumn{2}{|c|}{ nd } & \multicolumn{2}{|c|}{ wc } & \multicolumn{2}{|c|}{ nd } & \multicolumn{2}{|c|}{ wc } & \multicolumn{2}{|c|}{ nd } \\
\hline & PP & PI & PP & PI & PP & PI & PP & PI & PP & PI & PP & PI \\
\hline $\mathrm{N}$ & 23 & 23 & 20 & 20 & 37 & 37 & 38 & 38 & 41 & 41 & 47 & 47 \\
\hline Chl & $0.50^{*}$ & $0.99 * * *$ & $\begin{array}{l}-0.03 \\
\end{array}$ & $-0.75 * * *$ & $-0.63 * * *$ & $0.99 * * *$ & $0.32^{(*)}$ & $-0.62 * * *$ & $0.36^{*}$ & 0.98 **** & $-0.74 * * *$ & $-0.81^{* * * *}$ \\
\hline Chl.a & 0.21 & $0.98 * * *$ & -0.18 & $-0.72 * * *$ & $-0.68^{* * *}$ & $0.99 * * *$ & $0.35^{*}$ & $-0.63 * * *$ & $0.38^{*}$ & $1.00 * * *$ & $-0.75^{* * *}$ & $-0.84^{* * * *}$ \\
\hline Chl.b & $0.40^{(*)}$ & $1.00^{* * *}$ & -0.09 & $-0.76^{* * * *}$ & $-0.64 * * *$ & $0.99 * * *$ & $0.31^{(*)}$ & $-0.62 * * *$ & $0.33^{*}$ & $0.97 * * *$ & $-0.75^{* * *}$ & $-0.78^{* * * *}$ \\
\hline Pheo.a & $0.76^{* * *}$ & $0.89 * * *$ & 0.07 & $-0.66 * *$ & $-0.33 *$ & $0.70 * * *$ & 0.16 & $-0.44 * *$ & $0.28^{(*)}$ & $0.68 * * *$ & $-0.45 * *$ & $-0.51^{* * * *}$ \\
\hline Chl.a : Chl & $-0.91 * * *$ & -0.12 & -0.24 & 0.33 & $-0.77 * * *$ & $0.79 * * *$ & $0.46 * *$ & $-0.69 * * *$ & -0.14 & 0.06 & -0.07 & -0.17 \\
\hline Chl.b : Chl & -0.17 & $0.76 * * *$ & -0.14 & $-0.41^{(*)}$ & $-0.57 * * *$ & $0.54 * * *$ & 0.11 & $-0.30^{(*)}$ & $-0.47 * *$ & $-0.32 *$ & 0.09 & $0.62 * * *$ \\
\hline Pheo.a : Chl & $0.85^{* * *}$ & -0.03 & 0.24 & -0.25 & $0.80 * * *$ & $-0.82 * * *$ & $-0.46 * *$ & $0.70 * * *$ & 0.13 & 0.14 & 0.05 & 0.04 \\
\hline
\end{tabular}

Significances are marked in bold. Dark and light grey shadows highlight the most relevant relationships for wc and nd spectra characteristics, respectively

${ }^{*} p \leq 0.1 ; *^{*} \leq 0.05 ; * * \leq 0.01 ; * * * \leq 0.001$ (significances of correlation coefficient $R$ ) 
Comparison of Spectral Characteristics in Nondestructively and Wet Chemically Derived Spectra

Having shown reasonable explanations for the pattern found in the extract spectra, the link to the unimodal pattern found in intact fruits still needs to be evaluated. The total pigment contents are well reflected in peak intensity, both in the fruit extract (wc) and - although less strong - in the intact fruit (nd) spectra (Table 1). This is also indicated by the significant, same-directed correlations between peak intensity values of wc and nd spectra in all three fruit species $\left(R_{\mathrm{A}}=-0.77, n=20\right.$; $R_{\mathrm{M}}=-0.60, n=37 ; R_{\mathrm{T}}=-0.81, n=41 ; p<0.001$ for all).

The peak position values of the nondestructively obtained spectra in apple and tomato were not linearly correlated to those of the destructively measured ones. However, for apple and tomato samples capturing a wide range of fruit development, the observed shifts in nd and wc peak positions follow a similar pattern: A-in the nondestructive spectra pronounced - right shift from the 'high' chlorophyll content class to the next (two) lower class(es) is followed by a-this time in the wet chemical spectra stronger pronounced - shift in the opposite direction (Fig. 2; middle and lower row). The mango peak positions behave differently: Peak positions in the nondestructive and wet chemical spectra are negatively correlated $\left(R_{\mathrm{M}}=-0.41, p=0.011 ;\right.$ Fig. 2$)$. For this difference among the fruit species, no clear explanation exists at present. However, the more qualitative information on pigment ratios is better linked to the peak position of the fruit extract (wc) spectra (Table 1).

Other (molecular, environmental) changes of the chlorophyll molecule influencing its spectral properties could also influence the nondestructive spectrum. Further studies are needed to address these aspects.

\section{Conclusions}

The newly introduced apparent peak position of the red band chlorophyll absorption has the potential to serve as a spectral marker describing the ripening stage of a fruit more precisely than the commonly used peak intensity alone. High correlations were found between the peak position and the relative proportions of chlorophyll and its catabolites in destructive reference analyses. This offers an interesting and nondestructively available view on the underlying physiological processes during fruit maturation. However, for a comprehensive interpretation of nondestructively measured variations regarding the peak position, changes in the pigment environment (molecular bonds, polarity) need also be considered. Nevertheless, this approach might stimulate the scientific interest on nondestructive readings predicting the ripeness of fruit and vegetables.
Acknowledgments We thank Corinna Rolleczek, Gabriele Wegner, Sascha Kaufmann, Christian Regen and Ingo Truppel for their valuable lab assistance. We are grateful to Dennis Dannehl, Humboldt University Berlin, for providing the tomato samples. Part of this work was supported by the ICT-AGRI project '3D-Mosaic-Advanced Monitoring of Tree Crops for Optimized Management-How to Cope with Variability in Soil and Plant Properties?' which is funded by the European Commission's ERA-NET scheme under the 7th Framework Programme for Research (ICT-AGRI 95-Zude; 2810ERA095).

Open Access This article is distributed under the terms of the Creative Commons Attribution License which permits any use, distribution, and reproduction in any medium, provided the original author(s) and the source are credited.

\section{References}

Aiamla-or, S., Nakajima, T., Shigyo, M., \& Yamauchi, N. (2012). Pheophytinase activity and gene expression of chlorophylldegrading enzymes relating to UV-B treatment in postharvest broccoli (Brassica oleracea L. Italica Group) florets. Postharvest Biology and Technology, 63, 60-66.

Ajila, C. M., Bhat, S. G., \& Prasada Rao, U. J. S. (2007). Valuable components of raw and ripe peels from two Indian mango varieties. Food Chemistry, 102, 1006-1011.

Aziz, N. A. A., Wong, L. M., Bhat, R., \& Cheng, L. H. (2012). Evaluation of processed green and ripe mango peel and pulp flours (Mangifera indica var. Chokanan) in terms of chemical composition, antioxidant compounds and functional properties. Journal of the Science of Food and Agriculture, 92, 557-563.

Barry, C. S. (2009). The stay-green revolution: recent progress in deciphering the mechanisms of chlorophyll degradation in higher plants. Plant Science, 176, 325-333.

Cara, B., \& Giovannoni, J. J. (2008). Molecular biology of ethylene during tomato fruit development and maturation. Plant Science, $175,106-113$.

Eijckelhoff, C., \& Dekker, J. P. (1995). Determination of the pigment stoichiometry of the photochemical reaction center of photosystem II. Biochimica et Biophysica Acta (BBA) - Bioenergetics, 1231, 21-28.

Eijckelhoff, C., \& Dekker, J. P. (1997). A routine method to determine the chlorophyll alpha, pheophytin alpha and beta-carotene contents of isolated Photosystem II reaction center complexes. Photosynthesis Research, 52, 69-73.

Fish, W. W., Perkins-Veazie, P., \& Collins, J. K. (2002). A quantitative assay for lycopene that utilizes reduced volumes of organic solvents. Journal of Food Composition and Analysis, 15, 309-317.

Fraser, P. D., Truesdale, M. R., Bird, C. R., Schuch, W., \& Bramley, P. M. (1994). Carotenoid biosynthesis during tomato fruit development. (Evidence for tissue-specific gene expression). Plant Physiology, $105(1), 405-413$.

Freiman, Z. E., Rodov, V., Yablovitz, Z., Horev, B., \& Flaishman, M. A. (2012). Preharvest application of 1-methylcyclopropene inhibits ripening and improves keeping quality of 'Brown Turkey' figs (Ficus carica L.). Scientia Horticulturae, 138, 266-272.

Fry, S. C. (2003). Postharvest physiology | ripening. In B. Thomas (Ed.), Encyclopedia of Applied Plant Sciences (pp. 794-807). Oxford, UK: Elsevier.

Gautier, H., Diakou-Verdin, V., Benard, C., Reich, M., Buret, M., Bourgaud, F., et al. (2008). How does tomato quality (sugar, acid, and nutritional quality) vary with ripening stage, temperature, and irradiance? Journal of Agricultural and Food Chemistry, 56, 1241-1250.

Giovannoni, J. J. (2004). Genetic regulation of fruit development and ripening. The Plant Cell Online, 16, S170-S180. 
Gross, J. (1987). Pigments of fruit. Series: food science and technology. Oxford, UK: Academic.

Hörtensteiner, S., \& Kräutler, B. (2011). Chlorophyll breakdown in higher plants. Biochimica et Biophysica Acta, 1807, 977-988.

Janave, M. T., \& Sharma, A. (2006). Inhibition of chlorophyll degradation in stay-green Langra mango (Mangifera indica L.) fruits. BARC Newsletter, 273, 80-86.

Kienzle, S., Sruamsiri, P., Carle, R., Sirisakulwat, S., Spreer, W., \& Neidhart, S. (2012). Harvest maturity detection for 'Nam Dokmai \#4' mango fruit (Mangifera indica L.) in consideration of long supply chains. Postharvest Biology and Technology, 72, 64-75.

Knee, M. (1972). Anthocyanin, carotenoid and chlorophyll changes in peel of Cox Orange Pippin apples during ripening on and off-tree. Journal of Experimental Botany, 23(74), 184-196.

Kozukue, N., \& Friedman, M. (2003). Tomatine, chlorophyll, $\beta$-carotene and lycopene content in tomatoes during growth and maturation. Journal of the Science of food and Agriculture, 83, 195-200.

Kuckenberg, J., Tartachnyk, I., \& Noga, G. (2008). Evaluation of fluorescence and remission techniques for monitoring changes in peel chlorophyll and internal fruit characteristics in sunlit and shaded sides of apple fruit during shelf-life. Postharvest Biology and Technology, 48, 231-241.

Lang, C., \& Hübert, T. (2012). A colour ripeness indicator for apples. Food and Bioprocess Technology, 5, 3244-3249.

Lichtenthaler, H. K. (1987). Chlorophylls and carotenoids - pigments of photosynthetic biomembranes. Methods in Enzymology, 148, 350-382.

Lichtenthaler, H. K., \& Buschmann, C. (2001). Chlorophylls and carotenoids: Measurement and characterization by UV-VIS spectroscopy. Current Protocols in Food Analytical Chemistry (pp. F4.3.1-F4.3.8.). New York: Wiley.

Merzlyak, M. N., Solovchenko, A. E., \& Gitelson, A. A. (2003). Reflectance spectral features and non-destructive estimation of chlorophyll, carotenoid and anthocyanin content in apple fruit. Postharvest Biology and Technology, 27(2), 197-211.

Nattuvetty, V. R., \& Chen, P. (1980). Maturity sorting of green tomatoes based on light transmittance through regions of the fruit. Transactions of ASAE, 23(2), 515-518.

Nogueira, S. B., Labate, C. A., Gozzo, F. C., Pilau, E. J., Lajolo, F. M., \& Oliveira do Nascimento, J. R. (2012). Proteomic analysis of papaya fruit ripening using 2DE-DIGE. Journal of Proteomics, 75, 1428-1439.

OECD (1992) International standardisation of fruit and vegetables. Colour gauge for use by the trade in gauging the skin colouring of tomatoes (51 9205 3). Copyright CBT, The Netherlands. ISBN 8851-92053-0.
Pereira, L. F. P., Galvão, R. M., Kobayashi, A. K., Cação, S. M., \& Vieira, L. G. E. (2005). Ethylene production and acc oxidase gene expression during fruit ripening of Coffea arabica L. Brazilian Journal of Plant Physiology, 17, 283-289.

Pflanz, M., \& Zude, M. (2008). Spectrophotometric analyses of chlorophyll and single carotenoids during fruit development of tomato (Solanum lycopersicum L.) by means of iterative multiple linear regression analysis. Applied Optics, 47(32), 5961-5970.

Pilkington, S. M., Montefiori, M., Jameson, P. E., \& Allan, A. C. (2012). The control of chlorophyll levels in maturing kiwifruit. Planta, 236, $1615-1628$.

Saga, Y., \& Tamiaki, H. (2012). Demetalation of chlorophyll pigments. Chemistry \& Biodiversity, 9, 1659-1683.

Schelbert, S., Aubry, S., Burla, B., Agne, B., Kessler, F., Krupinska, K., et al. (2009). Pheophytin pheophorbide hydrolase (pheophytinase) is involved in chlorophyll breakdown during leaf senescence in Arabidopsis. Plant Cell, 21, 767-785.

Scott, K. J. (2001). Detection and measurements of carotenoids by UV/ VIS spectrophotometry. Current Protocols in Food Analytical Chemistry (pp F2.2.1-F2.2.10). John Wiley \& Sons, Inc.

Solovchenko, A. E., Kozhina, L., Nazarov, Y., \& Gudkovsky, V. A. (2011). Relationships between internal ethylene and optical reflectance in ripening 'Antonovka' apples grown under sunlit and shaded conditions. Postharvest Biology and Technology, 59, 206209.

White, R. C., Jones, I. D., \& Gibbs, E. L. E. A. (1963). Determination of chlorophylls, chlorophyllides, pheophytins, and pheophorbides in plant material. Journal of Food Science, 28, 431-436.

Xie, L. J., Ying, Y. B., \& Ying, T. J. (2009). Rapid determination of ethylene content in tomatoes using visible and short-wave nearinfrared spectroscopy and wavelength selection. Chemometrics and Intelligent Laboratory Systems, 97, 141-145.

Ziosi, V., Noferini, M., Fiori, G., Tadiello, A., Trainotti, L., Casadoro, G., et al. (2008). A new index based on vis spectroscopy to characterize the progression of ripening in peach fruit. Postharvest Biology and Technology, 49, 319-329.

Zude, M. (2003). Comparison of indices and multivariate models to nondestructively predict the fruit chlorophyll by means of visible spectrometry in apple fruit. Analytica Chimica Acta, 481(1), 119126.

Zude, M., \& Herold, B. (2002). Optimum harvest date determination for apples using spectral analysis. Gartenbauwissenschaft, 67(5), 199204. 